\title{
CORRECTIONS
}

\section{Union urges Spanish doctors to report effects of budget cuts on patient care}

In this News story by Aser García Rada (BMJ 2012;344:e1270, doi:10.1136/bmj.e1270) our translation of the Organización Médica Colegial as Spanish Medical Association was inaccurate. It is in fact the national association of Spanish medical colleges, and therefore the headline should have read "Medical Colleges
Association urges Spanish doctors to report effects of budget cuts on patient care."

Cite this as: BMJ 2012;344:e1749

๑ BMJ Publishing Group Ltd 2012 